\title{
Near-field sound radiation of fan tones from an installed turbofan aero-engine
}

\author{
Alan McAlpine, James Gaffney, and Michael J. Kingan \\ Institute of Sound and Vibration Research, University of Southampton, Southampton, Hampshire SO17 1BJ, United Kingdom
}

(Dated: 9 July 2015)

\begin{abstract}
The development of a distributed source model to predict fan tone noise levels of an installed turbofan aeroengine is reported. The key objective is to examine a canonical problem: how to predict the pressure field due to a distributed source located near an infinite, rigid cylinder. This canonical problem is a simple representation of an installed turbofan, where the distributed source is based on the pressure pattern generated by a spinning duct mode, and the rigid cylinder represents an aircraft fuselage. The radiation of fan tones can be modelled in terms of spinning modes. In this analysis, based on duct modes, theoretical expressions for the near-field acoustic pressures on the cylinder, or at the same locations without the cylinder, have been formulated. Simulations of the near-field acoustic pressures are compared against measurements obtained from a fan rig test. Also the installation effect is quantified by calculating the difference in the sound pressure levels with and without the adjacent cylindrical fuselage. Results are shown for the blade passing frequency fan tone radiated at a supersonic fan operating condition.
\end{abstract}

PACS numbers:

\section{INTRODUCTION}

Fan tones are a key noise source from turbofan aeroengines. Advanced theoretical and numerical methods to predict fan tones are routinely used by industry. Noise generated by the ducted fan in a turbofan engine is often described in terms of duct modes. Calculations of modes can be used to predict the in-duct sound propagation, and the sound radiation from the duct, albeit the majority of theoretical and numerical schemes predict the sound radiated into free space. Fewer schemes include installation acoustics in their modelling, that is how the radiated sound field is altered when the source, i.e. the aircraft engine, is installed on an aeroplane. In this article, the relevance of the work is directly linked to the issue of cabin noise in turbofan aircraft, specifically on the prediction of the sound pressure levels on the fuselage due to fan tones.

The key objective of this work is to formulate a canonical problem in order to study near-field installation acoustics. The basic set-up is shown sketched in Fig. 1. The objective is to predict the pressure on the surface of a rigid, cylindrical fuselage adjacent to a nearby distributed sound source. The distributed source is based on the pressure pattern generated by a spinning mode in a cylindrical duct. This is used to model a tone generated by a ducted fan. The prediction method will be used to evaluate the sound pressure levels on the surface of a cylindrical fuselage owing to specific rotor-alone tones generated by a supersonic ducted fan. These tones are harmonics of the engine's shaft rotation frequency and are the major contributor to what is known as the "buzz-saw" noise.

A simple modelling approach is proposed in order to predict the acoustic pressure in the near field. The predictions are based on calculating the radiation of a spinning mode from a cylindrical duct. A convenient approach in this case is the Rayleigh integral formulation, originally used by Tyler and Sofrin ${ }^{1}$. The Rayleigh integral is formulated by integrating infinitesimal volume sources located on the duct termination. The source strength of the volume sources (volume velocity) is proportional to the axial particle velocity at the duct termination. The Rayleigh integral can be used with a non-uniform velocity distribution across the duct termination, so the radi-

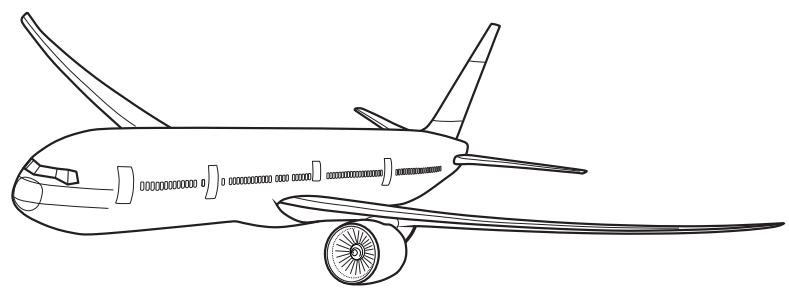

(a)

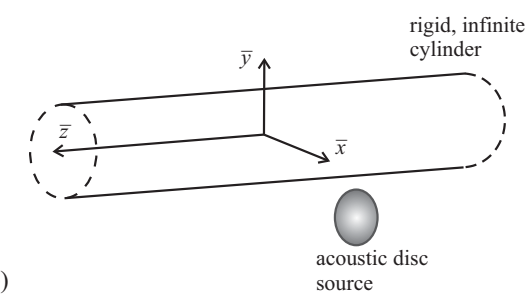

FIG. 1. (a) Sketch of an installed turbofan aero-engine. (b) Canonical problem for fan tone radiation from an installed turbofan aeroengine.

ation of spinning modes can be calculated, including taking account of clockwise or counter-clockwise spinning modes.

It is appropriate to only use the Rayleigh integral to calculate the sound radiation in the forward hemisphere. It is known that with an unflanged duct the predicted pressure field will not be realistic near sideline (polar angle equal to $90^{\circ}$ ). Hoctor $^{2}$ compared the directivity patterns for spinning modes radiated from a cylindrical duct, calculated using the WienerHopf technique (unflanged duct) and using the Rayleigh integral approach. He found that for practical applications the agreement between the two methods was good, at least up to polar angles around $70^{\circ}$.

Although the Rayleigh integral can be used to calculate the radiation of spinning modes from a cylindrical duct, it is not straightforward to include the effect of having a cylindrical fuselage in the near field. The approach used here is to combine the Rayleigh integral formulation with the theoretical solution for the pressure on a cylinder's surface due to 
a monopole point source located near the cylinder. In the absence of mean flow, the generic solution to this canonical problem is detailed in the text on Electromagnetic and Acoustic Scattering by Simple Shapes by Bowman, Senior and Uslenghi ${ }^{3}$, published in 1969 . In the context of the aircraft interior noise problem, Fuller ${ }^{4}$ has investigated spherical acoustic waves impinging on a cylinder, and in this reference the pressure field on the cylinder's surface due to a monopole source is detailed.

This article is a continuation of the work in McAlpine and Kingan $^{5}$ which examined open rotors and installation acoustics effects in the far field. The methodology in this new article is similar, but now the pressure is calculated in the near field, specifically on the surface of an adjacent cylindrical fuselage, and the source is a fan tone generated by a ducted fan, instead of a tone generated by an open rotor. In Ref. ${ }^{5}$ there is a review of previous work on this type of installation acoustics problem. The key relevant previous work is the procedure outlined in Hanson and Magliozzi ${ }^{6}$. In Ref. ${ }^{6}$ the incident field due to a propeller noise source in free space is used to determine the total field in the presence of an aircraft fuselage which is modelled by an infinite, rigid cylinder. The incident field is given by near-field frequency-domain propeller source theory developed previously by Hanson. This solution was derived in a coordinate system centred on the propeller. In Ref. ${ }^{6}$ the authors show how to shift the solution to a coordinate system which is centred on the axis of the cylinder, using Graf's Addition theorem. They then combine this with an appropriate form of the scattered field (a sum of outward propagating cylindrical waves): the resultant sum of the incident and scattered fields gives the total pressure field. Their analysis also includes the effect of the fuselage boundary layer, and they match their analytical solutions to a numerical solution in the boundary layer.

The methodology used in the present work is essentially the same as that proposed by Hanson and Magliozzi, albeit the tonal source is from a ducted fan rather than a propeller, and the analysis does not include the fuselage boundary layer. The key step in Hanson and Magliozzi is transforming the cylindrical polar coordinate system, moving the origin from the centre of the source to the centre of the fuselage. These two coordinate systems are shown sketched in Fig. 2.

A field point can be defined in terms of the cylindrical polar coordinate system, $(r, \phi, z)$, centred on the distributed source, or alternatively, in terms of the cylindrical polar coordinate system, $(\bar{r}, \bar{\phi}, \bar{z})$, centred on the cylindrical fuselage. These two coordinate systems are used in the mathematical formulation which is outlined in $\S$ II. The theory is followed by validation results in $\S \mathrm{III}$, and a selection of illustrative results based on a realistic model-scale test case in $\S \mathrm{IV}$.

\section{MATHEMATICAL FORMULATION}

Consider a rigid duct, radius $a$. Field points inside the duct are specified by the cylindrical polar coordinate system $(r, \phi, z)$, where the $z$-axis is aligned with the duct centreline. The duct has an unflanged termination at $z=0$. Also inside and outside the duct there is a subsonic uniform mean flow, Mach number $M_{z}=U_{z} / c_{0}$, directed in the negative $z$-direction.

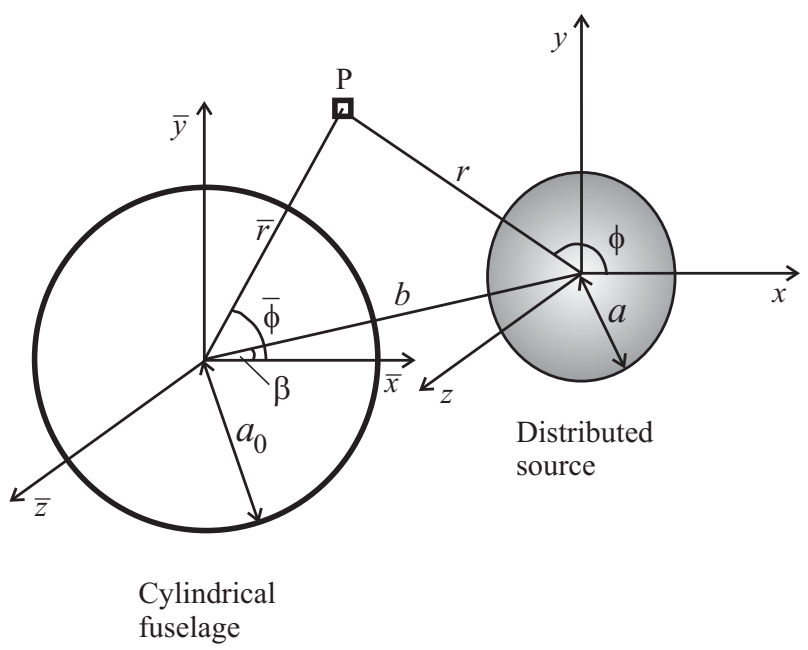

FIG. 2. Sketch of reference frames for the source and the cylinder.

\section{A. In-duct sound field}

The in-duct sound field is represented in terms of 'spinning modes'. Mode $(l, q)$ has azimuthal order $l$ and radial order $q$. Assuming time-harmonic waves, solving the convected Helmholtz equation, the acoustic pressure $p_{l q}^{\prime}=$ $\widehat{p}_{l q}(r, \phi, z) \exp \left\{\mathrm{i} \omega_{0} t\right\}$ and axial particle velocity $u_{z l q}=$ $\widehat{u}_{z l q}(r, \phi, z) \exp \left\{\mathrm{i} \omega_{0} t\right\}$ of mode $(l, q)$ are given by

$$
\begin{aligned}
\widehat{p}_{l q} & =P_{l q} \mathrm{~J}_{l}\left(\kappa_{l q} r\right) \mathrm{e}^{\mathrm{i}\left(-l \phi-k_{z l q} z\right)}, \\
\widehat{u}_{z l q} & =\frac{\xi_{l q}}{\rho_{0} c_{0}} P_{l q} \mathrm{~J}_{l}\left(\kappa_{l q} r\right) \mathrm{e}^{\mathrm{i}\left(-l \phi-k_{z l q} z\right)},
\end{aligned}
$$

where $P_{l q}$ is the modal amplitude,

$$
\xi_{l q}=\frac{k_{z l q}}{\left(k_{0}+k_{z l q} M_{z}\right)},
$$

and the dispersion relation is given by

$$
k_{z l q}^{2}+\kappa_{l q}^{2}=\left(k_{0}+k_{z l q} M_{z}\right)^{2}, \quad k_{0}=\omega_{0} / c_{0}>0 .
$$

Also $\mathrm{J}_{l}(\cdot)$ denotes the Bessel function of the first kind order $l,\left\{\kappa_{l q}: q=1,2,3, \ldots\right\}$ is the set of radial eigenvalues which satisfy $\mathrm{J}_{l}^{\prime}\left(\kappa_{l q} a\right)=0$ (which determines for given azimuthal order $l$ the radial modes of order $q$ in a rigid-walled duct of radius $a), k_{z l q}$ is the axial wavenumber of mode $(l, q), c_{0}$ is the speed of sound, and $\rho_{0}$ is the mean density of the fluid (air) inside the duct. Also, the modal sound power $W_{l q}$ is proportional to the square of the modal amplitude, i.e.

$$
\begin{gathered}
W_{l q}=\frac{\left|P_{l q}\right|^{2}}{2 \rho_{0} c_{0}} \pi\left[a^{2}-\left(\frac{l}{\kappa_{l q}}\right)^{2}\right] J_{l}^{2}\left(\kappa_{l q} a\right) \times \\
{\left[\left(1+M_{z}^{2}\right) \operatorname{Re}\left\{\xi_{l q}\right\}-M_{z}\left(1+\left|\xi_{l q}\right|^{2}\right)\right] .}
\end{gathered}
$$

\section{B. Incident field}

The acoustic pressure owing to a fan tone radiated from the duct is termed the incident field $p_{i}^{\prime}$. This field is determined by 


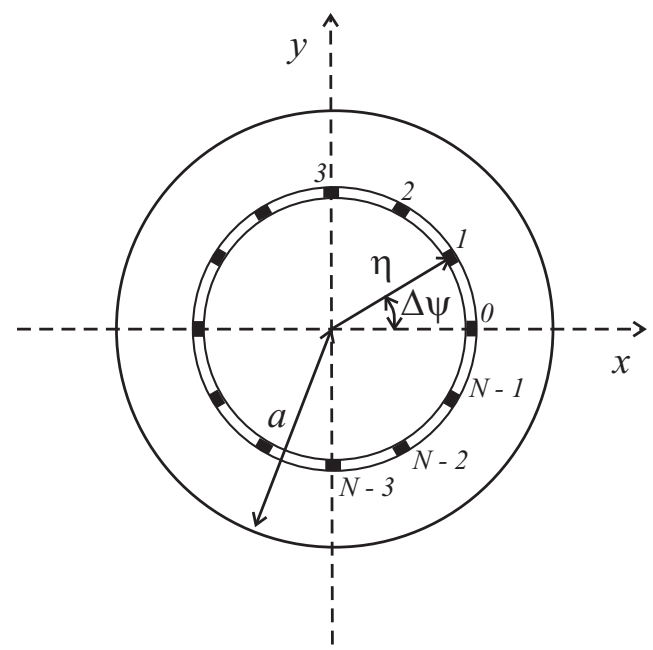

FIG. 3. Sketch of ring source at the duct termination.

integrating a distribution of monopole sources over the crosssection of the duct termination. This neglects the effect of diffraction by the edge of the duct.

First consider an annular ring source, comprised of $N$ monopoles distributed evenly around a circle of radius $\eta$. The point sources are numbered, $s=0,1,2, \ldots, N-1$. Monopole $s$ is located at $(r, \phi, z)=(\eta, s \Delta \psi, 0)$, where $\Delta \psi=2 \pi / N$. The annular ring source at the duct termination is shown sketched in Fig. 3.

A derivation of the incident pressure field owing to a rotating point source with angular velocity $\Omega$, centred at the origin and moving in the plane $z=0$, in the presence of subsonic uniform mean flow directed in the negative $z$-direction, is detailed in McAlpine and Kingan ${ }^{5}$. Equations (20-22) in Ref. ${ }^{5}$ give the incident field for a rotating monopole source. On setting $\Omega=0$, the field for a stationary point source is recovered. Accordingly, the pressure field owing to monopole source $s=0$ is given by

$$
\begin{aligned}
p_{i}^{\prime(0)}(r, \phi, z, t)= & \frac{1}{(2 \pi)^{2}} \sum_{m=-\infty}^{\infty}\{ \\
& \left.\left(\int_{-\infty}^{\infty} \overline{p_{i_{m}}^{\prime(0)}} \mathrm{e}^{-\mathrm{i} k_{z} z} \mathrm{~d} k_{z}\right) e^{-\mathrm{i} m \phi}\right\},
\end{aligned}
$$

where

$$
\begin{aligned}
\overline{p_{i_{m}}^{(0)}}\left(r, k_{z}, t\right)= & \frac{\pi}{2} Q^{(0)} \rho_{0} c_{0}\left(k_{0}+k_{z} M_{z}\right) \times \\
& \mathrm{H}_{m}^{(2)}\left(\Gamma_{0} r_{>}\right) \mathrm{J}_{m}\left(\Gamma_{0} r_{<}\right) \mathrm{e}^{\mathrm{i} \omega_{0} t},
\end{aligned}
$$

and the overbar is used to denote a variable which has been Fourier transformed.

The volume velocity of the monopole source is given by $Q^{(0)} \exp \left\{\mathrm{i} \omega_{0} t\right\}$. The variables $r_{>}$and $r_{<}$are defined as follows:

$$
\begin{aligned}
& r_{>}=\left\{\begin{array}{ll}
r, & r>\eta \\
\eta, & r<\eta
\end{array} ;\right. \\
& r_{<}=\left\{\begin{array}{ll}
\eta, & r>\eta \\
r, & r<\eta
\end{array} .\right.
\end{aligned}
$$

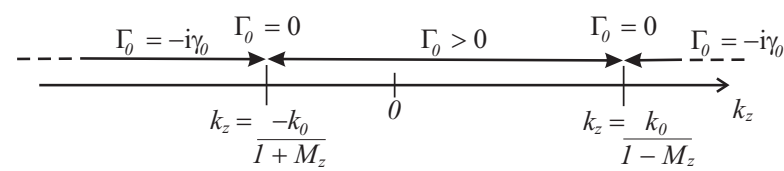

FIG. 4. Relationship between the axial wavenumber $k_{z}$ and the 'radial' wavenumber $\Gamma_{0}$.

Also the 'radial' wavenumber $\Gamma_{0}$ is given by

$$
\Gamma_{0}{ }^{2}=\left(k_{0}+k_{z} M_{z}\right)^{2}-k_{z}^{2} .
$$

In order to ensure that only outward propagating waves are allowed as $r \rightarrow \infty$, the appropriate values of $\Gamma_{0}$, as a function of $k_{z}$, are shown in Fig. 4 . In the range,

$$
\frac{-k_{0}}{1+M_{z}}<k_{z}<\frac{k_{0}}{1-M_{z}},
$$

$\Gamma_{0}$ is real, whilst outside this range $\Gamma_{0}=-\mathrm{i} \gamma_{0}$ where $\gamma_{0}=$ $\sqrt{k_{z}^{2}-\left(k_{0}+k_{z} M_{z}\right)^{2}}>0$.

Introduce the new angular coordinate $\widetilde{\phi}$ such that point source $s$ is located at $\widetilde{\phi}=0$. Then

$$
\widetilde{\phi}=\phi-s \Delta \psi,
$$

and from Equations $(6,7)$

$$
\begin{aligned}
& p_{i}^{\prime(s)}(r, \phi, z, t)=\frac{1}{(2 \pi)^{2}} \sum_{m=-\infty}^{\infty}\{ \\
& \left.\left(\int_{-\infty}^{\infty} \overline{p_{i_{m}}^{\prime(s)}} \mathrm{e}^{-\mathrm{i} k_{z} z} \mathrm{~d} k_{z}\right) e^{-\mathrm{i} m \tilde{\phi}}\right\} \\
& =\frac{Q^{(s)} \rho_{0} c_{0}}{8 \pi} \sum_{m=-\infty}^{\infty}\{ \\
& \left(\int_{-\infty}^{\infty}\left(k_{0}+k_{z} M_{z}\right) \mathrm{H}_{m}^{(2)}\left(\Gamma_{0} r_{>}\right) \times\right. \\
& \left.\mathrm{J}_{m}\left(\Gamma_{0} r_{<}\right) \mathrm{e}^{-\mathrm{i} k_{z} z} \mathrm{~d} k_{z}\right) \times \\
& \left.\mathrm{e}^{-\mathrm{i} m(\phi-s \Delta \psi)} \mathrm{e}^{\mathrm{i} \omega_{0} t}\right\} .
\end{aligned}
$$

The source strength $Q^{(s)}$ is specified by taking the axial particle velocity of spinning mode $(l, q)$ at $(r, \phi, z)=$ $(\eta, s \Delta \psi, 0)$. From Equation (2)

$$
\begin{aligned}
Q^{(s)} & =\widehat{u}_{z l q}(\eta, s \Delta \psi, 0) \delta A, \\
& =\frac{\xi_{l q} P_{l q}}{\rho_{0} c_{0}} \mathrm{~J}_{l}\left(\kappa_{l q} \eta\right) \mathrm{e}^{-\mathrm{i} l s \Delta \psi} \delta A,
\end{aligned}
$$

where $\delta A=\eta \delta \eta \delta \psi$.

The total incident field is given by

$$
p_{i}^{\prime}(r, \phi, z, t)=\sum_{s=0}^{N-1} p_{i}^{\prime(s)}(r, \phi, z, t) .
$$

Thus, combining Equations (14), (16) and (17) gives

$$
p_{i}^{\prime}(r, \phi, z, t)=\frac{\xi_{l q} P_{l q}}{8 \pi} \sum_{s=0}^{N-1} \sum_{m=-\infty}^{\infty}\{
$$




$$
\begin{aligned}
& \left(\int_{-\infty}^{\infty}\left(k_{0}+k_{z} M_{z}\right) \mathrm{H}_{m}^{(2)}\left(\Gamma_{0} r_{>}\right) \times\right. \\
& \left.\mathrm{J}_{m}\left(\Gamma_{0} r_{<}\right) \mathrm{J}_{l}\left(\kappa_{l q} \eta\right) \mathrm{e}^{-\mathrm{i} k_{z} z} \mathrm{~d} k_{z}\right) \times \\
& \left.\mathrm{e}^{-\mathrm{i}(l-m) s \Delta \psi} e^{-\mathrm{i} m \phi} \mathrm{e}^{\mathrm{i} \omega_{0} t} \eta \delta \eta \delta \psi\right\}
\end{aligned}
$$

The summation over $s$ can be found using the standard formula for a geometric progression. The summation is zero unless $m=l-n N$ where $n$ is an integer. Hence Equation (18) reduces to

$$
\begin{aligned}
p_{i}^{\prime}(r, \phi, z, t)= & N \frac{\xi_{l q} P_{l q}}{8 \pi} \sum_{n=-\infty}^{\infty}\{ \\
& \left(\int_{-\infty}^{\infty}\left(k_{0}+k_{z} M_{z}\right) \mathrm{H}_{l-n N}^{(2)}\left(\Gamma_{0} r_{>}\right) \times\right. \\
& \left.\mathrm{J}_{l-n N}\left(\Gamma_{0} r_{<}\right) \mathrm{J}_{l}\left(\kappa_{l q} \eta\right) \mathrm{e}^{-\mathrm{i} k_{z} z} \mathrm{~d} k_{z}\right) \times \\
& \left.\mathrm{e}^{\mathrm{i} n N \phi} \mathrm{e}^{-\mathrm{i} l \phi} \mathrm{e}^{\mathrm{i} \omega_{0} t} \eta \delta \eta \delta \psi\right\}
\end{aligned}
$$

Each ring source contains $N$ point sources. The distributed source is formed by taking the limit as $N \rightarrow \infty$, then $N \delta \psi \rightarrow$ $2 \pi$ and only the term $n=0$ is required in the summation, and also taking the limit as $\delta \eta \rightarrow 0$ and integrating from $\eta=0$ to $a$. This gives, at field points outside the duct termination $r>a$,

$$
\begin{aligned}
p_{i}^{\prime}(r, \phi, z, t)= & \frac{\xi_{l q} P_{l q}}{4} \int_{-\infty}^{\infty}\left(k_{0}+k_{z} M_{z}\right) \Psi_{l q} \times \\
& \mathrm{H}_{l}^{(2)}\left(\Gamma_{0} r\right) \mathrm{e}^{-\mathrm{i} k_{z} z} \mathrm{~d} k_{z} \mathrm{e}^{-\mathrm{i} l \phi} \mathrm{e}^{\mathrm{i} \omega_{0} t},
\end{aligned}
$$

where

$$
\Psi_{l q}=\int_{\eta=0}^{a} \mathrm{~J}_{l}\left(\Gamma_{0} \eta\right) \mathrm{J}_{l}\left(\kappa_{l q} \eta\right) \eta \mathrm{d} \eta
$$

The function $\Psi_{l}$ can be evaluated exactly. For non-plane-wave excitation:

$$
\begin{array}{ll}
\Psi_{l q}=\frac{\Gamma_{0} a}{\kappa_{l q}^{2}-\Gamma_{0}^{2}} \mathrm{~J}_{l}\left(\kappa_{l q} a\right) \mathrm{J}_{l}^{\prime}\left(\Gamma_{0} a\right), & \Gamma_{0} \neq \kappa_{l q}, \\
\Psi_{l q}=\frac{1}{2}\left(a^{2}-\frac{l^{2}}{\kappa_{l q}^{2}}\right) \mathrm{J}_{l}^{2}\left(\kappa_{l q} a\right), & \Gamma_{0}=\kappa_{l q} .
\end{array}
$$

Graf's Addition theorem is listed by Abramowitz and Stegun $^{7}$. Given the triangle shown sketched in Fig. 5, with sides of length $u, v$ and $w$, and angles $\alpha$ (between sides $u$ and $v$ ) and $\chi$ (between sides $u$ and $w$ ), then the theorem states that

$$
\zeta_{m}(w) \mathrm{e}^{\mathrm{i} m \chi}=\sum_{n=-\infty}^{\infty} \zeta_{m+n}(u) \mathrm{J}_{n}(v) \mathrm{e}^{\mathrm{i} n \alpha}
$$

where $\zeta_{m}$ denotes any of the Bessel functions $\mathrm{J}_{m}, \mathrm{Y}_{m}, \mathrm{H}_{m}^{(1)}$, $\mathrm{H}_{m}^{(2)}$, or linear combinations thereof. If the variables are complex, it is also required that $\left|v \mathrm{e}^{ \pm \mathrm{i} \alpha}\right|<|u|$, unless $\zeta_{m}=\mathrm{J}_{m}$.

Graf's Addition theorem is now used to transform the incident field to the cylindrical polar coordinate system $(\bar{r}, \bar{\phi}, \bar{z})$ centred on the cylindrical fuselage as shown in Fig. 2. It is emphasised that the cylinder has not yet been included in the

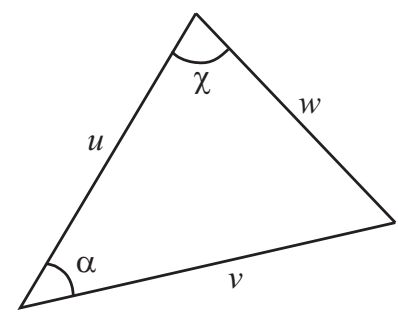

FIG. 5. Graf's Addition theorem.

modelling. At this step in the analysis, the aim is to find the incident pressure field at $\bar{r}=a_{0}$, which will be the location of the surface of the cylinder (when it is included in the subsequent analysis). Graf's addition theorem can be used for transforming between the coordinate systems $(r, \phi, z)$ and $(\bar{r}, \bar{\phi}, \bar{z})$.

Specifically, at the field point $r>a$ and $\bar{r}<b$, set $u=\Gamma_{0} b$, $v=\Gamma_{0} \bar{r}, w=\Gamma_{0} r, \alpha=\bar{\phi}-\beta$ and $\chi=\pi+\beta-\phi$. Note that $\bar{r}<b \Rightarrow|v|<|u|$ (as $\alpha$ is real). Then direct application of Graf's Addition theorem leads to

$$
\begin{aligned}
\mathrm{H}_{l}^{(2)}\left(\Gamma_{0} r\right) e^{-\mathrm{i} l \phi}= & \sum_{n=-\infty}^{\infty}\left\{(-1)^{(l+n)} \mathrm{H}_{l-n}^{(2)}\left(\Gamma_{0} b\right) \times\right. \\
& \left.\mathrm{J}_{n}\left(\Gamma_{0} \bar{r}\right) \mathrm{e}^{-\mathrm{i} n \bar{\phi}} \mathrm{e}^{-\mathrm{i}(l-n) \beta}\right\} .
\end{aligned}
$$

Now substitute Equation (25) into Equation (20) to find the incident field. Expressing this in the form of Equations $(6,7)$ gives

$$
\begin{aligned}
p_{i}^{\prime}(\bar{r}, \bar{\phi}, \bar{z}, t)= & \frac{1}{(2 \pi)^{2}} \sum_{n=-\infty}^{\infty}\{ \\
& \left.\left(\int_{-\infty}^{\infty} \overline{p_{i n}^{\prime}} \mathrm{e}^{-\mathrm{i} k_{z} \bar{z}} \mathrm{~d} k_{z}\right) e^{-\mathrm{i} n \bar{\phi}}\right\},
\end{aligned}
$$

where

$$
\begin{aligned}
\overline{p_{i n}^{\prime}}\left(\bar{r}, k_{z}, t\right)= & \pi^{2} \xi_{l q} P_{l q}(-1)^{l+n} \mathrm{e}^{-\mathrm{i}(l-n) \beta}\left(k_{0}+k_{z} M_{z}\right) \times \\
& \Psi_{l q} \mathrm{H}_{l-n}^{(2)}\left(\Gamma_{0} b\right) \mathrm{J}_{n}\left(\Gamma_{0} \bar{r}\right) \mathrm{e}^{\mathrm{i} \omega_{0} t} .
\end{aligned}
$$

\section{Scattered field}

Since the scattering object is an infinite cylinder, the scattered acoustic waves will be outward propagating cylindrical waves. The scattered field is given by

$$
\begin{aligned}
p_{s}^{\prime}(\bar{r}, \bar{\phi}, \bar{z}, t)= & \frac{1}{(2 \pi)^{2}} \sum_{n=-\infty}^{\infty}\{ \\
& \left.\left(\int_{-\infty}^{\infty} \overline{p_{s n}^{\prime}} \mathrm{e}^{-\mathrm{i} k_{z} \bar{z}} \mathrm{~d} k_{z}\right) e^{-\mathrm{i} n \bar{\phi}}\right\},
\end{aligned}
$$

where

$$
\overline{p_{s n}^{\prime}}\left(\bar{r}, k_{z}, t\right)=\frac{1}{2 \pi} \int_{-\infty}^{\infty} A_{n}\left(k_{z}, \omega\right) \mathrm{H}_{n}^{(2)}(\Gamma \bar{r}) \mathrm{e}^{\mathrm{i} \omega t} \mathrm{~d} \omega,
$$

and $\Gamma$ is given by Equation (10) with $k_{0}$ replaced by $k=\omega / c_{0}$. 
The total pressure field $p_{t}^{\prime}$ due to the distributed source located adjacent to an infinite, rigid cylinder is given by

$$
p_{t}^{\prime}=p_{i}^{\prime}+p_{s}^{\prime}
$$

where the total field satisfies a rigid wall boundary condition on the surface of the cylinder, i.e.

$$
\frac{\partial p_{t}^{\prime}}{\partial \bar{r}}=0 \quad \text { at } \quad \bar{r}=a_{0}
$$

Taking the Fourier transform leads to

$$
\frac{\partial \overline{p_{t n}^{\prime}}}{\partial \bar{r}}=\frac{\partial \overline{p_{i n}^{\prime}}}{\partial \bar{r}}+\frac{\partial \overline{p_{s n}^{\prime}}}{\partial \bar{r}}=0 \quad \text { at } \quad \bar{r}=a_{0}
$$

On substituting Equations (27) and (29) into the boundary condition (32) this gives

$$
\begin{aligned}
& \left(\pi^{2} \xi_{l q} P_{l q}(-1)^{l+n} \mathrm{e}^{-\mathrm{i}(l-n) \beta}\left(k_{0}+k_{z} M_{z}\right) \Psi_{l q} \times\right. \\
& \left.\mathrm{H}_{l-n}^{(2)}\left(\Gamma_{0} b\right) \Gamma_{0} \mathrm{~J}_{n}^{\prime}\left(\Gamma_{0} a_{0}\right) \mathrm{e}^{\mathrm{i} \omega_{0} t}\right)+ \\
& \frac{1}{2 \pi} \int_{-\infty}^{\infty} A_{n}\left(k_{z}, \omega\right) \Gamma \mathrm{H}_{n}^{(2) \prime}\left(\Gamma a_{0}\right) \mathrm{e}^{\mathrm{i} \omega t} \mathrm{~d} \omega=0,
\end{aligned}
$$

where ' denotes differentiation with respect to the function's argument.

In order to solve Equation (33), set

$$
A_{n}\left(k_{z}, \omega\right)=B_{n}\left(k_{z}, \omega\right) \delta\left(\omega-\omega_{0}\right)
$$

where $\delta(\omega)$ is the Dirac-delta function. The result is

$$
\begin{aligned}
B_{n}\left(k_{z}, \omega_{0}\right)= & -2 \pi^{3} \xi_{l q} P_{l q}(-1)^{l+n} \mathrm{e}^{-\mathrm{i}(l-n) \beta} \times \\
& \left(k_{0}+k_{z} M_{z}\right) \Psi_{l q} \mathrm{H}_{l-n}^{(2)}\left(\Gamma_{0} b\right) \times \\
& \left(\mathrm{J}_{n}^{\prime}\left(\Gamma_{0} a_{0}\right) / \mathrm{H}_{n}^{(2) \prime}\left(\Gamma_{0} a_{0}\right)\right),
\end{aligned}
$$

and

$$
\begin{aligned}
\overline{p_{s n}^{\prime}}\left(\bar{r}, k_{z}, t\right)= & -\pi^{2} \xi_{l q} P_{l q}(-1)^{l+n} \mathrm{e}^{-\mathrm{i}(l-n) \beta} \times \\
& \left(k_{0}+k_{z} M_{z}\right) \Psi_{l q} \mathrm{H}_{l-n}^{(2)}\left(\Gamma_{0} b\right) \mathrm{H}_{n}^{(2)}\left(\Gamma_{0} \bar{r}\right) \times \\
& \left(\mathrm{J}_{n}^{\prime}\left(\Gamma_{0} a_{0}\right) / \mathrm{H}_{n}^{(2) \prime}\left(\Gamma_{0} a_{0}\right)\right) \mathrm{e}^{\mathrm{i} \omega_{0} t}
\end{aligned}
$$

\section{Total field}

The total pressure field $p_{t}^{\prime}$ is given by

$$
\begin{aligned}
p_{t}^{\prime}(\bar{r}, \bar{\phi}, \bar{z}, t)= & \frac{1}{(2 \pi)^{2}} \sum_{n=-\infty}^{\infty}\{ \\
& \left.\left(\int_{-\infty}^{\infty} \overline{p_{t n}^{\prime}} \mathrm{e}^{-\mathrm{i} k_{z} \bar{z}} \mathrm{~d} k_{z}\right) e^{-\mathrm{i} n \bar{\phi}}\right\},
\end{aligned}
$$

where

$$
{\overline{p_{t n}^{\prime}}}^{\prime}={\overline{p_{i n}^{\prime}}}^{\prime}{\overline{p_{s n}^{\prime}}}
$$

Summing Equations (27) and (36) gives

$$
\begin{aligned}
{\overline{p_{t n}^{\prime}}}\left(\bar{r}, k_{z}, t\right)= & \pi^{2} \xi_{l q} P_{l q}(-1)^{l+n} \mathrm{e}^{-\mathrm{i}(l-n) \beta} \times \\
& \left(k_{0}+k_{z} M_{z}\right) \Psi_{l q} \mathrm{H}_{l-n}^{(2)}\left(\Gamma_{0} b\right) \times \\
& {\left[\left(\mathrm{J}_{n}\left(\Gamma_{0} \bar{r}\right) \mathrm{H}_{n}^{(2) \prime}\left(\Gamma_{0} a_{0}\right)-\right.\right.} \\
& \left.\mathrm{J}_{n}^{\prime}\left(\Gamma_{0} a_{0}\right) \mathrm{H}_{n}^{(2)}\left(\Gamma_{0} \bar{r}\right)\right) / \\
& \left.\mathrm{H}_{n}^{(2) \prime}\left(\Gamma_{0} a_{0}\right)\right] \mathrm{e}^{\mathrm{i} \omega_{0} t} .
\end{aligned}
$$

On the surface of the cylinder $\bar{r}=a_{0}$. Using the Wronskian formula,

$$
\mathrm{J}_{n}(x) \mathrm{H}_{n}^{(2) \prime}(x)-\mathrm{J}_{n}^{\prime}(x) \mathrm{H}_{n}^{(2)}(x)=-\mathrm{i}\left(\frac{2}{\pi x}\right),
$$

leads to

$$
\begin{aligned}
\overline{p_{t n}^{\prime}}\left(a_{0}, k_{z}, t\right)= & \pi^{2} \xi_{l q} P_{l q}(-1)^{l+n} \mathrm{e}^{-\mathrm{i}(l-n) \beta} \times \\
& \left(k_{0}+k_{z} M_{z}\right) \Psi_{l q} \mathrm{H}_{l-n}^{(2)}\left(\Gamma_{0} b\right) \times \\
& {\left[\left(-\mathrm{i}\left(2 / \pi \Gamma_{0} a_{0}\right)\right)\right.} \\
& \left.\mathrm{H}_{n}^{(2) \prime}\left(\Gamma_{0} a_{0}\right)\right] \mathrm{e}^{\mathrm{i} \omega_{0} t} .
\end{aligned}
$$

Finally, the total pressure field on the surface of the cylinder is expressed in the form

$$
\begin{aligned}
p_{t}^{\prime}\left(a_{0}, \bar{\phi}, \bar{z}, t\right)= & \frac{\xi_{l q} P_{l q}}{4}(-1)^{l} \mathrm{e}^{-\mathrm{i} l \beta} \mathrm{e}^{\mathrm{i} \omega_{0} t} \sum_{n=-\infty}^{\infty}\{ \\
& \left.(-1)^{n} \mathcal{I}_{n}^{(\text {wc })}\left(a_{0}, \bar{z}\right) \mathrm{e}^{-\mathrm{i} n(\bar{\phi}-\beta)}\right\},
\end{aligned}
$$

where

$$
\begin{aligned}
\mathcal{I}_{n}^{(\text {wc })}\left(a_{0}, \bar{z}\right)= & \int_{-\infty}^{\infty}\left(k_{0}+k_{z} M_{z}\right) \Psi_{l q} \mathrm{H}_{l-n}^{(2)}\left(\Gamma_{0} b\right) \times \\
& {\left[\left(-\mathrm{i}\left(2 / \pi \Gamma_{0} a_{0}\right)\right) /\right.} \\
& \left.\mathrm{H}_{n}^{(2) \prime}\left(\Gamma_{0} a_{0}\right)\right] \mathrm{e}^{-\mathrm{i} k_{z} \bar{z}} \mathrm{~d} k_{z} .
\end{aligned}
$$

The superscript (wc) denotes 'with cylinder'.

By way of comparison, rearranging equations (26) and (27), the incident pressure field on $\bar{r}=a_{0}$, in the absence of the cylinder, can be expressed in the same form as Equation (42), by replacing $\mathcal{I}_{n}^{(\mathrm{wc})}$ with

$$
\begin{aligned}
\mathcal{I}_{n}^{(\mathrm{nc})}\left(a_{0}, \bar{z}\right)= & \int_{-\infty}^{\infty}\left(k_{0}+k_{z} M_{z}\right) \Psi_{l q} \mathrm{H}_{l-n}^{(2)}\left(\Gamma_{0} b\right) \times \\
& \mathrm{J}_{n}\left(\Gamma_{0} a_{0}\right) \mathrm{e}^{-\mathrm{i} k_{z} \bar{z}} \mathrm{~d} k_{z} .
\end{aligned}
$$

The superscript (nc) denotes 'no cylinder'.

Equations (42-44) are the key results derived from this new analysis.

\section{VALIDATION}

\section{A. Incident field}

In McAlpine and Kingan ${ }^{5}$, distributed source models are derived for an open rotor. These can be used to model either 
thickness noise or loading noise. In this work, a different form of distributed source is used to model fan tone noise for a turbofan engine. The distributed source is based on the pressure pattern for an individual spinning duct mode. Rotor-alone fan tones are suitable to model in terms of spinning modes. Other fan tones also can be modelled using multi-mode sources, provided that it is appropriate to sum these sources incoherently.

The calculation of the distributed source's near field neglects diffraction by the intake duct lip. (The duct itself is not included in the modelling.) As mentioned in the Introduction, Hoctor $^{2}$ has shown that for practical applications, the directivity patterns for spinning modes radiated from a cylindrical duct, calculated using the Wiener-Hopf technique (unflanged duct) and using the Rayleigh integral approach, should be in good agreement at least up to polar angles around $70^{\circ}$.

In Gabard and Astley ${ }^{8}$, a new Wiener-Hopf solution is derived for a time-harmonic spinning mode exiting an annular duct where there is a vortex sheet between the jet flow and the freestream. This solution also can be used for an intake duct (where there is no vortex sheet) taking uniform mean flow everywhere. In this case, the result is equivalent to the WienerHopf solution in Homicz and Lordi ${ }^{9}$. The code based on the solution in Gabard and Astley is named 'GXMunt'. A sketch of the problem set-up is shown in Fig. 6(a).

The amplitude of the incident mode is specified. The nearand/or far-field pressure can be evaluated using the GXMunt code. This method includes diffraction at the duct exit, albeit the duct wall is infinitely thin, so a realistic intake lip profile is not modelled. Also reflection at the duct termination is included in the method.

By comparison, a sketch of the problem set-up to calculate sound radiation using the distributed source is shown in Fig. 6(b). This method does not include diffraction at the duct exit. Also reflection at the duct termination is not included in the method. (This is because there is no duct.)

The purpose of this validation study is to assess whether the distributed source model can be used to accurately model a spinning mode exiting a cylindrical duct. A series of validation test cases have been carried out. The expectation is that the distributed source model will be appropriate to use for most modes, other than modes near cut off. This is because the principal radiation lobe, for a mode that is close to being cut-off, will be at polar angle close to $90^{\circ}$, so diffraction by the duct lip is likely to be more significant. In addition, more energy will be reflected by the duct termination for modes near cut off.

A selection of the results from the validation study are shown in Figs. $7 \& 8$. All the results are obtained in the near field at $R=1 \mathrm{~m}$. Polar directivity plots are shown, which include the predicted levels calculated by the two methods. Also an example of the radiated pressure field visualised on a hemispherical three-dimensional plot is shown.

Overall, the near-field pressures predicted by both methods are very similar. As expected, there are small discrepancies between the two methods, but these only occur at high polar angles greater than $\approx 75^{\circ}$. Also, the absolute levels are very similar, so for these test cases, reflections at the duct termination are weak, and can be neglected.

Although the Wiener-Hopf solution is based on a more realistic model of sound radiation from an intake duct, this solution is far less amenable to use to specify the incident field

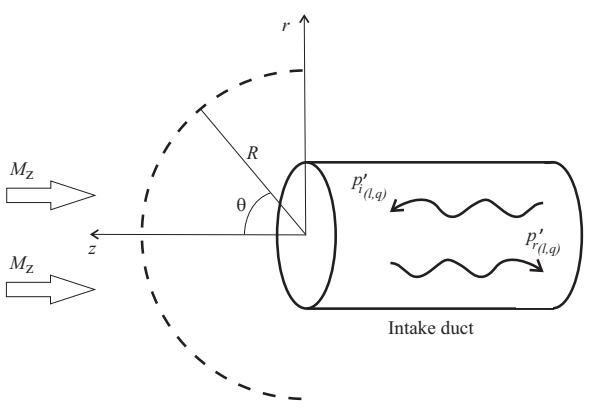

(a)

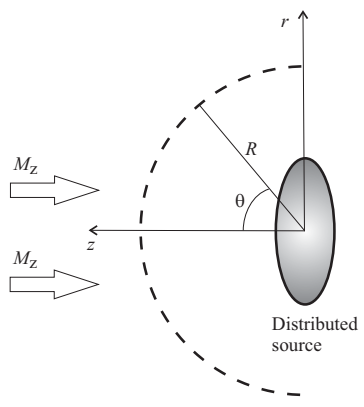

(b)

FIG. 6. (a) Problem set-up for the Wiener-Hopf solution. (b) Problem set-up for the distributed source solution.
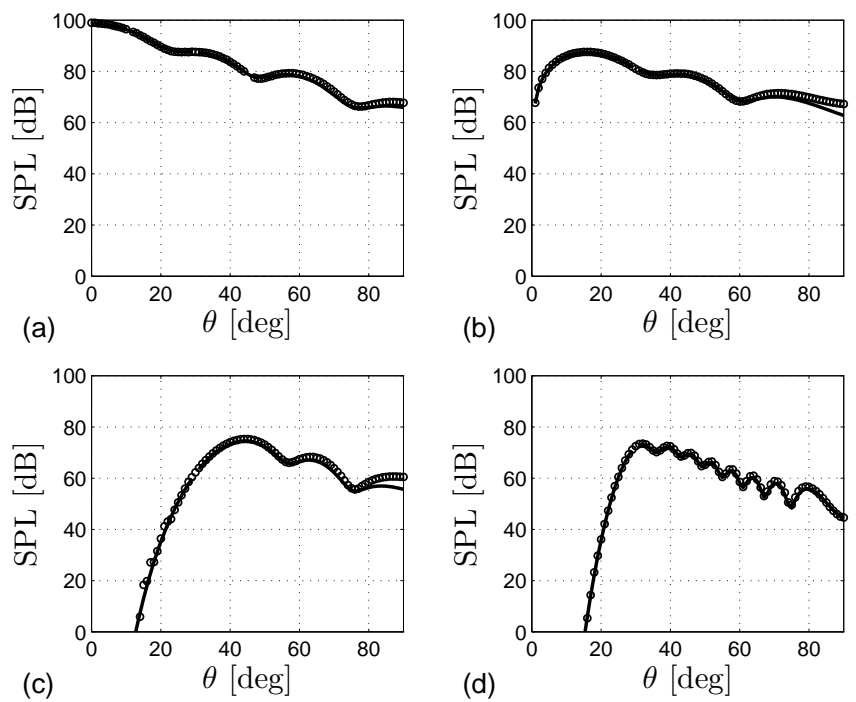

FIG. 7. Polar directivity at $R=1 \mathrm{~m}$. Comparison between solutions for a spinning duct mode (-) and a distributed source (o): (a) Mode $(0,1), f=1000 \mathrm{~Hz}, M_{z}=0.5$; (b) Mode $(1,1), f=1000 \mathrm{~Hz}$, $M_{z}=0.5$; (c) Mode $(10,1), f=2000 \mathrm{~Hz}, M_{z}=0.5$; (d) Mode $(20,1), f=5000 \mathrm{~Hz}, M_{z}=0.5$.

in order to calculate the near field in the presence of a cylindrical fuselage. The distributed source model provides a very similar prediction of the incident field in the near field, compared to the Wiener-Hopf solution, and this solution is far more amenable to use to calculate the near field in the presence of a cylindrical fuselage. 


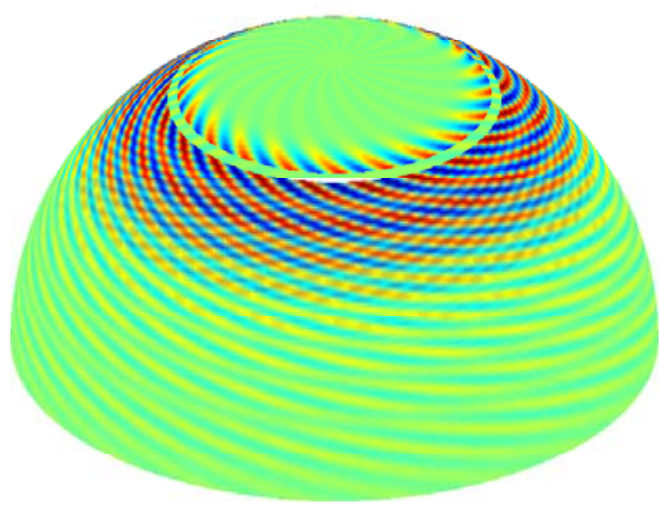

(a) Spinning mode.

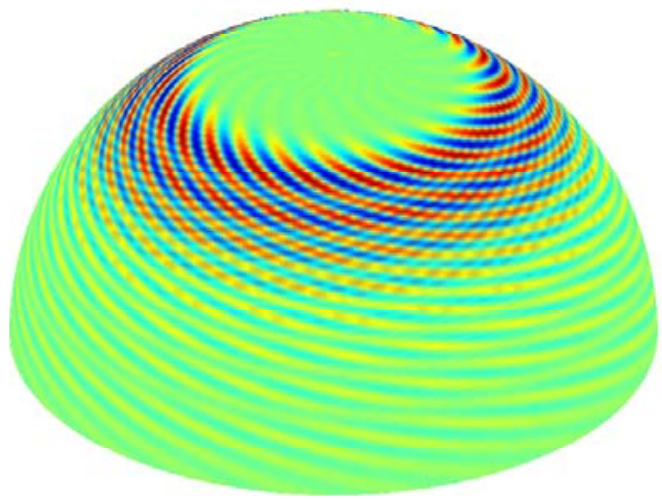

(b) Distributed source.

FIG. 8. Real part of pressure field at $R=1 \mathrm{~m}$. Mode $(20,1), f=$ $5000 \mathrm{~Hz}, M_{z}=0.5$. (Color online)

\section{B. Scattered field}

The implementation of the cylinder scattering model is verified by comparing the results for a distributed source with a reference solution. In this case, an appropriate reference solution is the scattered field due to a monopole source adjacent to a rigid cylinder which has been published previously by Fuller ${ }^{4}$.

In the reference solution, the distributed source shown in Fig. 2 is replaced by a stationary monopole point source located at the origin of the coordinate system $(x, y, z)$. Using the nomenclature employed in this article, the total pressure on the surface of the cylinder, due to a monopole source with volume velocity $Q_{0} \exp \left\{\mathrm{i} \omega_{0} t\right\}$, can be expressed in the form

$$
\begin{aligned}
p_{t}^{\prime}\left(a_{0}, \bar{\phi}, \bar{z}, t\right)= & \frac{Q_{0} \rho_{0} c_{0}}{8 \pi} \mathrm{e}^{\mathrm{i} \omega_{0} t} \sum_{n=-\infty}^{\infty}\left\{\mathcal{I}_{n_{\text {mono }}}^{(\text {wc })}\left(a_{0}, \bar{z}\right) \times\right. \\
& \left.\mathrm{e}^{-\mathrm{i} n(\bar{\phi}-\beta)}\right\},
\end{aligned}
$$

where

$$
\begin{aligned}
\mathcal{I}_{n_{\text {mono }}}^{(\mathrm{wc})}\left(a_{0}, \bar{z}\right)= & \int_{-\infty}^{\infty}\left(k_{0}+k_{z} M_{z}\right) \mathrm{H}_{n}^{(2)}\left(\Gamma_{0} b\right) \times \\
& {\left[\left(-\mathrm{i}\left(2 / \pi \Gamma_{0} a_{0}\right)\right) /\right.} \\
& \left.\mathrm{H}_{n}^{(2) \prime}\left(\Gamma_{0} a_{0}\right)\right] \mathrm{e}^{-\mathrm{i} k_{z} \bar{z}} \mathrm{~d} k_{z} .
\end{aligned}
$$

Also the incident pressure field on $\bar{r}=a_{0}$, in the absence of the cylinder, can be expressed in the same form as Equation (45), by replacing $\mathcal{I}_{n \text { mono }}^{(\text {wc })}$ with

$$
\begin{aligned}
\mathcal{I}_{n_{\text {mono }}}^{(\mathrm{nc})}\left(a_{0}, \bar{z}\right)= & \int_{-\infty}^{\infty}\left(k_{0}+k_{z} M_{z}\right) \mathrm{H}_{n}^{(2)}\left(\Gamma_{0} b\right) \times \\
& \mathrm{J}_{n}\left(\Gamma_{0} a_{0}\right) \mathrm{e}^{-\mathrm{i} k_{z} \bar{z}} \mathrm{~d} k_{z} .
\end{aligned}
$$

Equations (45-47) can be obtained from the solutions for a rotating monopole source detailed in McAlpine and Kingan ${ }^{5}$. Equations $(36,37)$ in Ref. ${ }^{5}$, on taking $\bar{r}=a_{0}$, give the total pressure field for a rotating monopole source on the surface of the cylinder. On setting $\Omega=0$, the field for a stationary point source is recovered. However, this source is located at $(x, y, z)=(a, 0,0)$, so also it is necessary to set $a=0$, which will eliminate the summation in Equation (37) in Ref. ${ }^{5}$, and the expression for $p_{t}^{\prime}\left(a_{0}, \bar{\phi}, \bar{z}, t\right)$ reduces to Equations $(45,46)$. In order to obtain Equation (47), use Equation (25) instead of Equation (37) in Ref. ${ }^{5}$, which is the appropriate equation for the incident pressure field for a rotating monopole source, expressed in the coordinate system $(\bar{r}, \bar{\phi}, \bar{z})$ centred on the cylindrical fuselage.

Fuller ${ }^{4}$ has examined the effect of spherical acoustic waves impinging on a rigid cylinder in the absence of any mean flow. In order to assess the cylinder scattering and blockage effects, Fuller defined the 'scattering correction factor'

$$
\alpha_{c}=20 \log _{10}\left(1+\left.\frac{p_{s}^{\prime}}{p_{i}^{\prime}}\right|_{\bar{r}=a_{0}}\right)=20 \log _{10}\left(\left.\frac{p_{t}^{\prime}}{p_{i}^{\prime}}\right|_{\bar{r}=a_{0}}\right) .
$$

In Figure 2 from Ref. ${ }^{4}$, the values of the correction factor $\alpha_{c}$ for a monopole source located adjacent to a rigid cylinder are plotted for four examples of low frequency sources, with Helmholtz number $k a_{0}=0.5,1.0,2.0$ and 4.0. The monopole is located at $\bar{x} / a_{0}=2, \bar{y} / a_{0}=0$ and $\bar{z} / a_{0}=0$. The correction factor is calculated in the range, $\bar{\phi}=0$ to $180^{\circ}$, in the source plane $\bar{z} / a_{0}=0$.

Data points from Figure 2 in Fuller ${ }^{4}$ are reproduced in Fig. 9. The symbols $(\circ),(\triangle),(\square)$ and $(\times)$ show the previously published data from Ref. ${ }^{4}$. At Helmholtz number $k a_{0}=0.5,1.0,2.0$ and 4.0, the published results are compared against predictions of $\alpha_{c}$ computed by evaluating Equations (45-47). The purpose of this comparison is to verify that the computation of the scattered field generated by a source adjacent to a rigid cylinder has been properly implemented. In this case, with a monopole source, it is seen in Fig. 9 that the pressure field on the cylinder has been accurately computed since the computed values of $\alpha_{c}$ are in very good agreement with the data from Ref. ${ }^{4}$.

Results of $\alpha_{c}$ computed by evaluating Equations (42-44) for a small distributed source are compared against the results for the monopole source at Helmholtz number $k a_{0}=0.5,1.0$, 2.0 and 4.0 in Fig. 10. The example is the same as shown in Fig. 9, and in Figure 2 in Fuller ${ }^{4}$, except now $\alpha_{c}$ has been computed for a distributed source adjacent to a rigid cylinder. The distributed source has radius $a / a_{0}=0.01$, which is very small, thus the Helmholtz number of the distributed source $k a<<1$, and the source radiation is approximately the same as the radiation from a monopole point source. This result confirms that the computation of the scattered field generated 


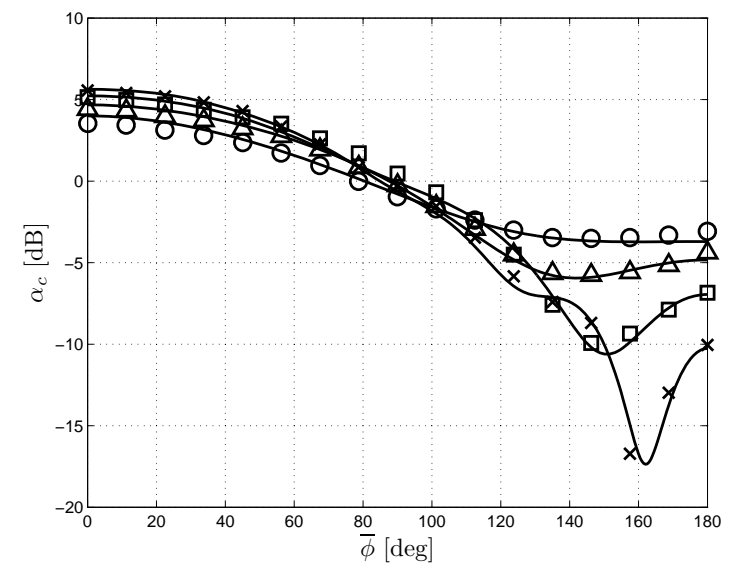

FIG. 9. Scattering correction factor $\alpha_{c}$ (48) vs. azimuthal angle $\bar{\phi}$ in the source plane $\bar{z} / a_{0}=0$ for a monopole source located at $\bar{x} / a_{0}=$ 2 and $\bar{y} / a_{0}=0$ and with zero mean flow. The symbols show data points taken from Figure 2 in Fuller ${ }^{4}:(\circ) k a_{0}=0.5,(\triangle) k a_{0}=$ 1.0, ( $\square) k a_{0}=2.0$ and $(\times) k a_{0}=4.0$. The solid lines are the simulation results from the monopole source code written to evaluate the pressure field on the surface of the cylinder, cf. Equations (4547).

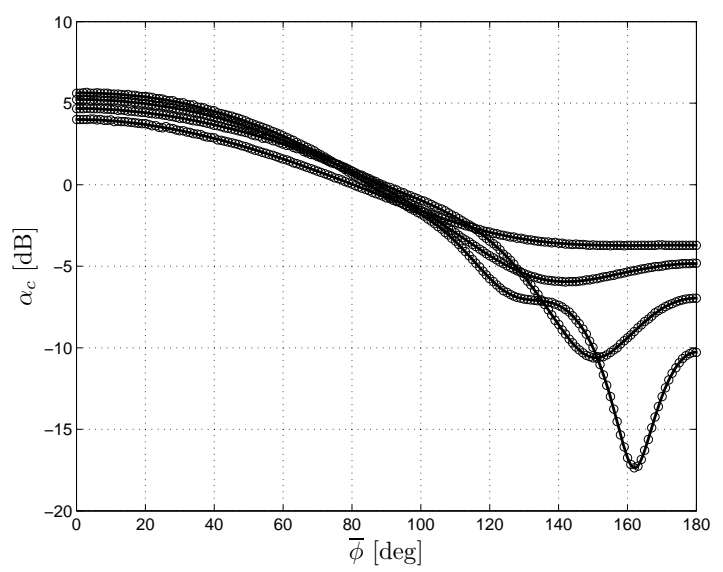

FIG. 10. Scattering correction factor $\alpha_{c}$ (48) vs. azimuthal angle $\bar{\phi}$ in the source plane $\bar{z} / a_{0}=0$ for a monopole source located at $\bar{x} / a_{0}=2$ and $\bar{y} / a_{0}=0$ and with zero mean flow. The solid lines are the simulation results for a monopole source with frequency $k a_{0}=0.5,1.0,2.0$ and 4.0 (see Fig. 9 for details). Also included are the simulation results for a distributed source (o) with small radius $a / a_{0}=0.01$.

by a distributed source adjacent to a rigid cylinder has been properly implemented.

\section{Convergence study}

In $\S \S I I I . B$ it is seen that the pressure fields due to a monopole or distributed source adjacent to a rigid cylinder are comparable at very low Helmholtz numbers. It is interesting to examine the mathematical solution for the pressure field on the surface of the cylinder due to a distributed source $(42,43)$, in the limit as the source's radius $a \rightarrow 0$. In this limit, all the duct modes are cut-off except the plane wave mode $(l, q)=(0,1)$ for which $\kappa_{01}=0, k_{z 01}=k, \xi_{01}=1$ and $\hat{p}_{01} / \hat{u}_{z 01}=\rho_{0} c_{0}$ (refer to $\S$ II, $\S \S$ II.A, Equations (1-4)). In essence the distributed source is equivalent to a small piston vibrating time-harmonically.

The only term in Equations $(42,43)$ which is dependent on the source radius $a$ is the function $\Psi_{l q}(21)$. It can be shown that

$$
\Psi_{01}= \begin{cases}\frac{1}{2} a^{2}+\mathcal{O}\left(\left(\Gamma_{0} a\right)^{3}\right), & \Gamma_{0}>0 \\ \frac{1}{2} a^{2}+\mathcal{O}\left(\left(\gamma_{0} a\right)^{3}\right), & \Gamma_{0}=-\mathrm{i} \gamma_{0}, \gamma_{0}>0\end{cases}
$$

Then it is straightforward to show that Equations $(42,43)$ reduce to Equations $(45,46)$ on setting

$$
\Psi_{01}=\frac{1}{2} a^{2} \quad \text { and } \quad \frac{P_{01}}{\rho_{0} c_{0}}\left(\pi a^{2}\right)=Q_{0},
$$

the latter being equivalent to Equation (16). This reduces the solution for the pressure field on the surface of the cylinder due to a distributed source to that of the solution for the monopole source.

However, replacing $\Psi_{01}$ by $\frac{1}{2} a^{2}$ is only valid if terms of $\mathcal{O}\left(\left(\Gamma_{0} a\right)^{3}\right)$ and $\mathcal{O}\left(\left(\gamma_{0} a\right)^{3}\right)$ are negligible. Evaluation of $\mathcal{I}_{n}^{(\text {wc) }}$ (43) involves integration over $k_{z}$. As seen in Figure 4 , in the range $-k_{0} /\left(1+M_{z}\right)<k_{z}<k_{0} /\left(1-M_{z}\right)$ (cf. Equation 11), $\Gamma_{0}$ is real and positive. In this range, the maximum value of $\Gamma_{0}=k_{0} / \sqrt{1-M_{z}^{2}}$, so in the limit as $a \rightarrow 0$, terms of $\mathcal{O}\left(\left(\Gamma_{0} a\right)^{3}\right)$ will be negligible provided that $k_{0} a / \sqrt{1-M_{z}^{2}}<<1$.

However, outside this range, $\Gamma_{0}=-\mathrm{i} \gamma_{0}$ where $\gamma_{0}$ is real and positive. As $k_{z} \rightarrow \pm \infty$ then $\gamma_{0} \rightarrow\left|k_{z}\right| \sqrt{1-M_{z}^{2}}$ so it cannot be assumed that terms of $\mathcal{O}\left(\left(\gamma_{0} a\right)^{3}\right)$ are negligible in the limit as $a \rightarrow 0$ because $\gamma_{0}$ has no upper bound.

Thus, replacing $\Psi_{01}$ by $\frac{1}{2} a^{2}$ in Equation (43), in the limit as $a \rightarrow 0$, strictly is not permissible. However, in practice, finite integration limits, say $-K<k_{z}<K$, will be taken in order to evaluate the integral over $k_{z}$ using numerical integration. These limits can be determined by the asymptotic expansion of the Hankel function $\mathrm{H}_{n}^{(2)}$ for large argument. It can be shown that

$$
\frac{\mathrm{H}_{n}^{(2)}\left(\Gamma_{0} b\right)}{\mathrm{H}_{n}^{(2) \prime}\left(\Gamma_{0} a_{0}\right)} \sim e^{-\left|k_{z}\right| \sqrt{1-M_{z}^{2}}\left(b-a_{0}\right)} \text { as } k_{z} \rightarrow \pm \infty,
$$

and thus the integrand in Equation (43) will be exponentially small at sufficiently large $\left|k_{z}\right|$. The integration limits also will depend on $\left(b-a_{0}\right)$ which is the distance of the source from the nearest point on the cylinder's surface. Therefore, in practice, terms of $\mathcal{O}\left(\left(\gamma_{0} a\right)^{3}\right)$ will be negligible provided that $K a \sqrt{1-M_{z}^{2}}<<1$.

This brief analysis verifies that computation of the pressure field on the cylinder's surface for a small distributed source, at very low frequency, will be equivalent to the computation of the pressure field for a monopole source, provided that the radius $a$ of the disc is sufficiently small. Numerical verification of this result is shown in Figure 10.

Finally, as part of the validation, the convergence of the solutions for the incident and scattered fields are examined. The 

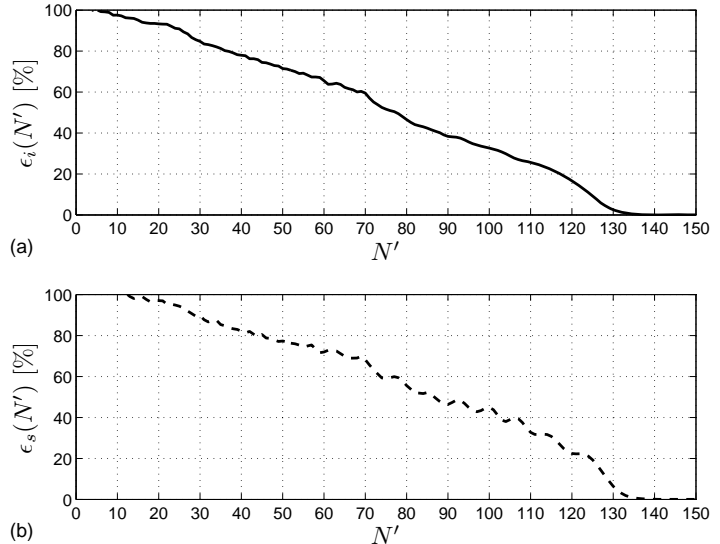

(b)

FIG. 11. Example of convergence for the simulation results from the distributed source code. The relative error $\epsilon$ (52) is shown in (a) for the incident field $p_{i}^{\prime}(-)$ and in (b) for the scattered field $p_{s}^{\prime}(---)$. In each case the converged solution is used as the reference value in Equation (52). The example is for mode $(20,1), f=5000 \mathrm{~Hz}$, $M_{z}=0.5, b / a_{0}=3, a / a_{0}=0.5, \beta=0^{\circ}$. (This validation test case also has been used in $\S$ III, $\S \S$ III.A, cf. Fig. 7(d) and Fig. 8.) The error is evaluated over the surface of the cylinder extending from $\bar{z} / a_{0}=0$ to 5 .

expressions both for the incident and scattered pressure fields, (26) and (28) respectively, involve infinite summations over the azimuthal harmonics $n$. These summations are truncated at the $N$ th harmonic, once it has been checked that the series has converged. The value of $N$ is dependent on the parameters of each specific calculation of the incident and scattered fields.

An example showing convergence of both the incident and scattered fields is shown in Fig. 11. Convergence is monitored via the average root mean square relative error, $\epsilon$, which is defined by

$$
\epsilon_{\square}\left(N^{\prime}\right)=\sqrt{\frac{1}{M} \sum^{M}\left|\frac{p_{\square_{N^{\prime}}}^{\prime}-p_{\square_{N}}^{\prime}}{p_{\square_{N}}^{\prime}}\right|^{2}},
$$

where the subscript $\square$ denotes either $i$ or $s$ for the incident or scattered pressure field respectively. In Equation (52) the subscript $N^{\prime}$ denotes the pressure field which has been calculated by summing up to the $N^{\prime}$ th harmonic only. The converged solution is reached when $N^{\prime} \rightarrow N$. Also the squared relative error has been averaged over all the grid points on the surface of the cylinder, where $M$ denotes the total number of grid points. The square root of this average gives the rms value of the relative error. It can be seen in Fig. 11 that the convergence rate of the incident and scattered fields are very similar, and in this example which is at a relatively high-frequency, it is necessary to include more than a hundred harmonics in order to obtain a converged solution.

\section{ILLUSTRATIVE RESULTS}

Simulation results are shown for two example model-scale test cases, in $\S \S$ IV.A with no cylinder in the near field, followed in $\S \S$ IV.B with the cylinder in the near field. A sketch

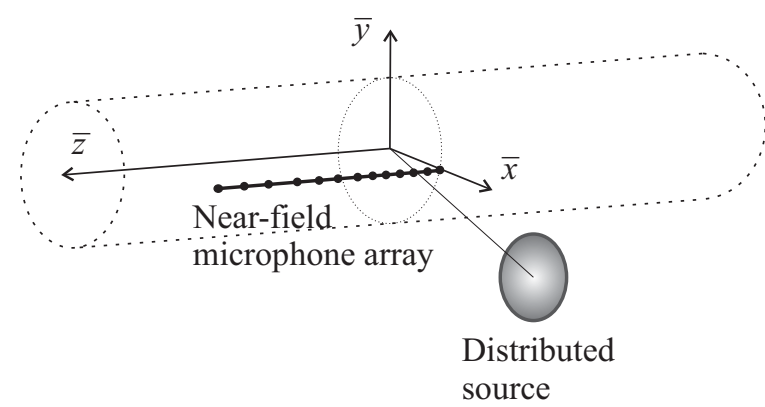

(a)

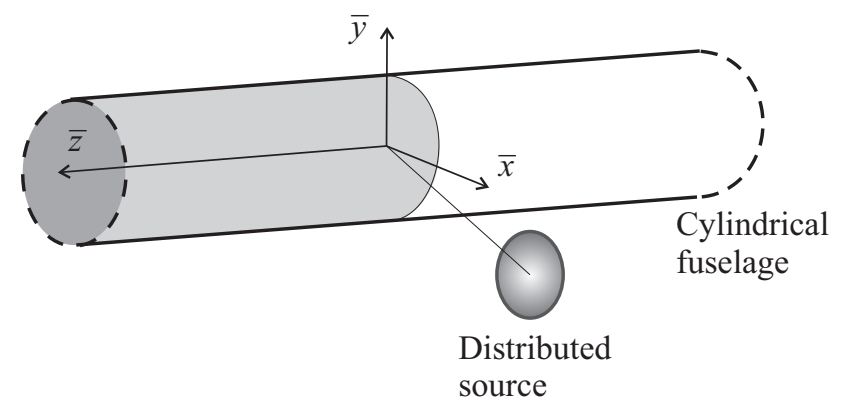

(b)

FIG. 12. (a) Set-up for model test case with no cylinder. (The location of the near-field microphone array is shown.) (b) Set-up for model test case with cylinder.

of the test case set-ups are shown in Fig. 12. In both test cases the same negative angle for $\beta$ is used (refer to Fig. 2 where the angle $\beta$ is defined).

\section{A. No cylinder}

An example of measurements from a static intake rig test conducted at the AneCom AeroTest facility are utilised to provide some illustrative results. The measurements were first published in Sugimoto et al. ${ }^{10}$ In this article, some measurements acquired by a near-field microphone array from Ref. ${ }^{10}$ are reproduced.

The model fan rig is around one-third full-scale. Sound produced by the fan propagates inside the model intake duct, and radiates into an anechoic chamber. Outside the intake the sound was measured by a linear near-field microphone array, and a polar far-field microphone array. No model-scale fuselage was used in the tests, but the near-field array was positioned roughly where an adjacent fuselage would be located, such that the microphones' positions were all forward of the fan plane in a linear array. A sketch of the experimental set-up is shown in Fig. 12(a).

The example results reproduced in this article are the measurements at blade passing frequency (BPF) at $90 \%$ fan speed (supersonic fan operating condition). At this frequency, in total around eighty modes are predicted to be cut-on, ranging from azimuthal order $l=-22$ to 22 . The sound field inside the intake was measured with a linear axial microphone array and also a circumferential mode detection array. The fan had twenty blades; hence the rotor-locked modal compo- 
nent of the BPF tone sound field will be predominantly mode $(l, q)=(20,1)$ since, at this high fan speed, the higher radial orders at azimuthal order $l=20$ are cut-off. Mode detection data is shown in Figure 3 in Ref. ${ }^{10}$. The rotor-locked mode, azimuthal order $l=20$, measured inside the lined intake, is around 15 to $20 \mathrm{~dB}$ higher compared to the non-rotor-locked azimuthal orders in the range $l=-22$ to 22 , which in turn are higher than the background noise floor. The level of the rotor-locked mode, compared to the non-rotor-locked modes, inside the unlined intake was much higher, but the value of the mode protrusion could not be determined because the dynamic range of the microphone array was not sufficient. However, as shown in Figure 4(a) in Ref. ${ }^{10}$, the measured BPF tone is at least $25 \mathrm{~dB}$ higher in the unlined intake compared to the lined intake duct. Assuming that most of the energy is contained in the rotor-locked mode, inside the unlined intake, an estimate of the rotor-locked mode protrusion is around $45 \mathrm{~dB}$ higher compared to the non-rotor-locked azimuthal orders.

Near-field array measurements compared with predictions are shown in Fig. 13. The near-field array data is reproduced from Figure 4(b) in Ref. ${ }^{10}$. The non-dimensional axial position is defined $Z=\bar{z} / a_{0}$. The first microphone in the linear array was located at $Z=0$, in the same plane as the termination (exit) of the intake duct. The rest of the microphones were all located upstream in the forward arc. Also the linear array is located at $\bar{\phi}=0$. The measurements of the sound pressure level (SPL) have been adjusted. For both measurement and prediction, the maximum SPL has been scaled to equal zero. Multi-mode predictions have been performed, whereby the radiated field of each cut-on mode is calculated, and then the SPL has been calculated by summing the modes incoherently.

In Fig. 13(a), the prediction on taking equal energy per mode is shown. (The link between the modal power and modal amplitude is given by Eq. (5).) Also shown are the measurements with the lined and unlined intake. It is evident that simply assuming equal energy per mode is not appropriate in this case. This is because, as discussed, at this high fan speed, the rotor-locked mode will be the principal component of the sound field. In Figs. 13(b) \& (c), the predicted results are for equal energy per mode, except the rotor-locked mode $(l, q)=(20,1)$. It's modal amplitude has been adjusted to include $+45 \mathrm{~dB}$ tone protrusion with the unlined intake, Fig. 13(b), or $+20 \mathrm{~dB}$ tone protrusion with the lined intake, Fig. 13(c). In both cases, the predictions show much closer agreement with the measured data.

Also shown are predictions for just the rotor-locked mode. With the unlined intake, the level of the rotor-locked tonal component decays rapidly with distance upstream. In the region $0<Z<2$ the rotor-locked mode is dominant, but further upstream in the region $Z>3$ it is the non-rotor-locked components of the sound field which are dominant. This is expected because the non-rotor-locked components of the sound field include modes which propagate close to the axis of the intake duct. With the lined intake, the level of the rotor-locked mode is lower, owing to attenuation by the lining, so the rotorlocked mode is dominant over a smaller upstream extent, in the region $0<Z<1$.

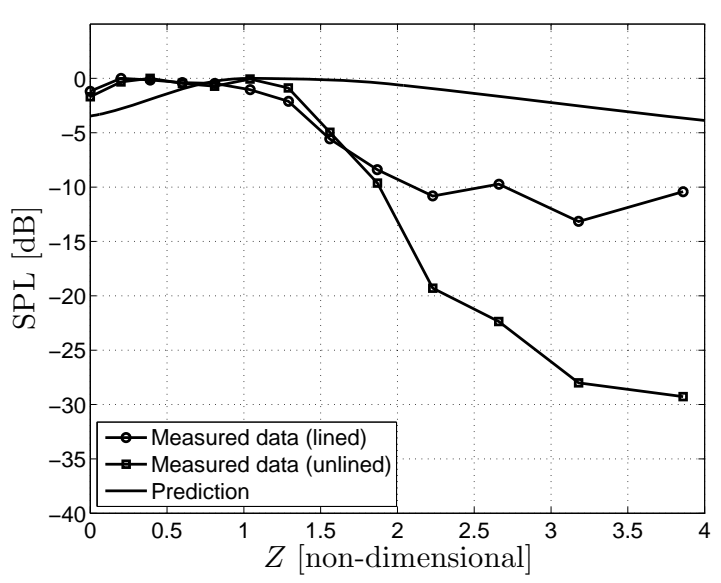

(a) Source $=$ equal energy per mode.

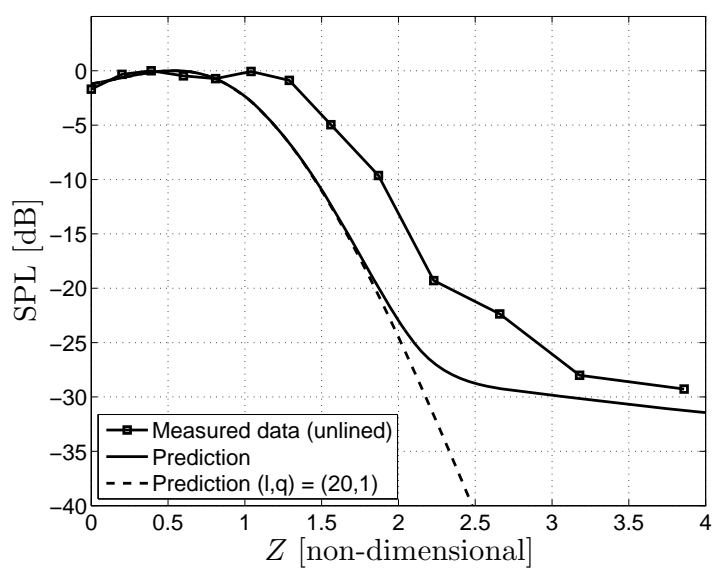

(b) Source $=$ equal energy per mode except rotor-locked mode $(l, q)=(20,1),+45 \mathrm{~dB}$ tone protrusion.

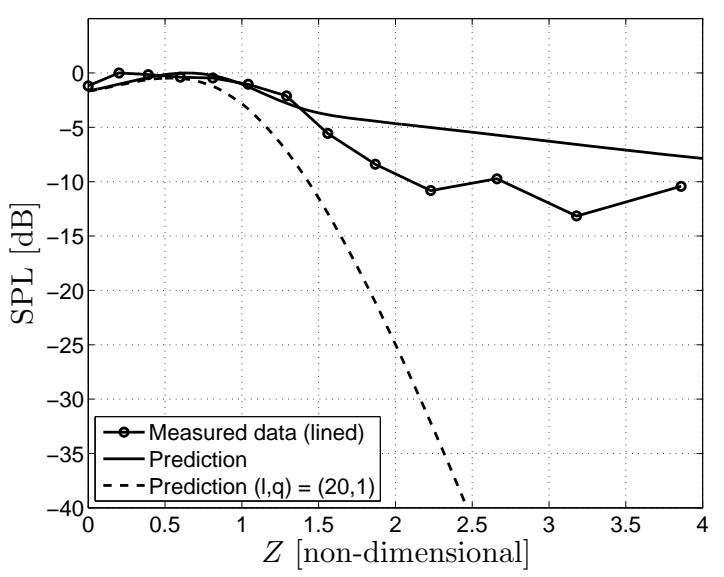

(c) Source $=$ equal energy per mode except rotor-locked mode $(l, q)=(20,1),+20 \mathrm{~dB}$ tone protrusion.

FIG. 13. Near-field array: Comparison of measurement and prediction at blade passing frequency, $90 \%$ fan speed. The experimental data includes measurements taken with an unlined and lined intake duct. 


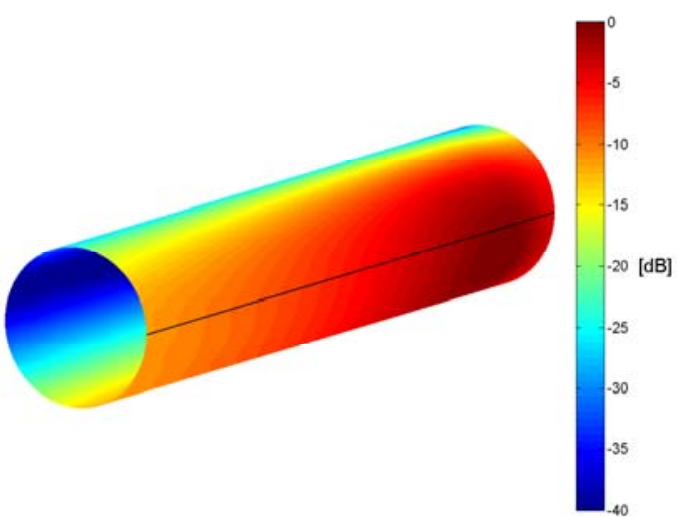

(a) Source $=$ equal energy per mode.

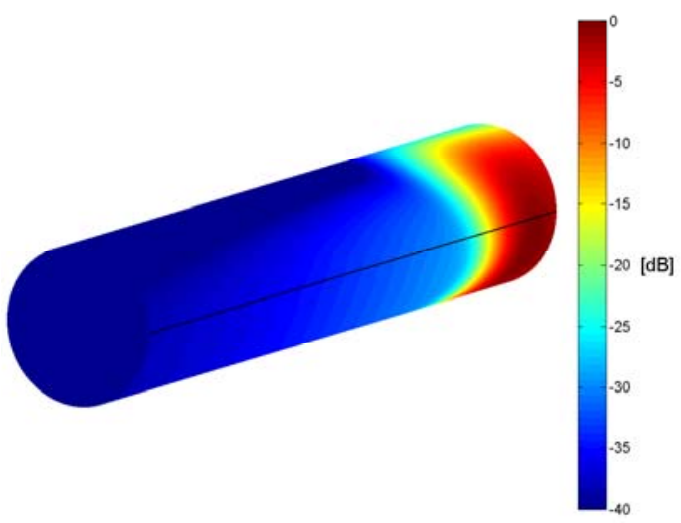

(b) Source $=$ equal energy per mode except rotor-locked mode $(l, q)=(20,1),+45 \mathrm{~dB}$ tone protrusion.

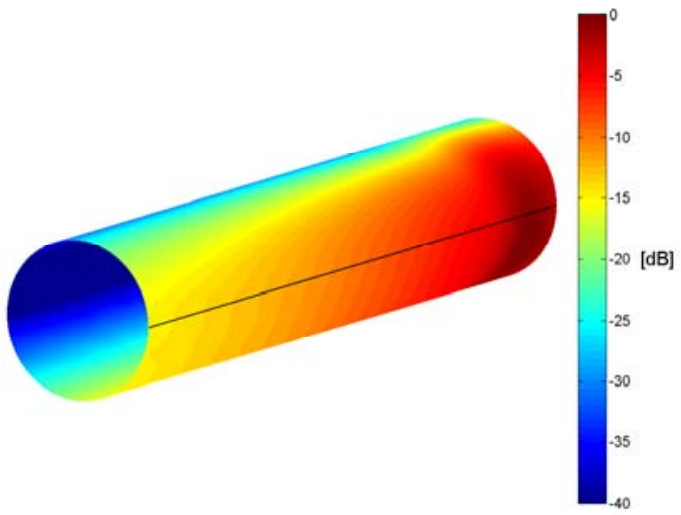

(c) Source $=$ equal energy per mode except rotor-locked mode $(l, q)=(20,1),+20 \mathrm{~dB}$ tone protrusion.

FIG. 14. Prediction of sound pressure level on the surface of the cylindrical fuselage. Simulation at blade passing frequency, $90 \%$ fan speed. The black solid line shows the location of $\bar{\phi}=0$. In each example the sound pressure levels have been adjusted to set the maximum value equal to zero. (Color online)

\section{B. With cylinder}

Illustrative examples showing SPL contours on a cylindrical fuselage are shown in Fig. 14. A sketch of the simulation set-up is shown in Fig. 12(b). In these examples, the pressure on the cylinder in the region $0<Z<10$ is shown. As before, the levels have been adjusted and the maximum SPL equals zero. The source models used for the simulations in Fig. 14 $(\mathrm{a}-\mathrm{c})$ correspond to the same source models used for the simulations in Fig. 13(a-c) respectively. Additionally in Fig. 15 the effect of the cylindrical fuselage is quantified by plotting contours of the difference in the sound pressure level with and without the cylinder. This difference is defined as

$$
\Delta=\mathrm{SPL}_{\text {with cylinder }}-\mathrm{SPL}_{\text {no cylinder }} .
$$

Only the contour plot of $\Delta$ for the equal energy per mode source is shown. The contour plots for the other two examples (including the effect of rotor-locked mode protrusion) are very similar.

In these examples, there is only one source region, so on the far side of the cylinder there is a shadow zone where the levels are much lower. It is seen in Fig. 15 that around $\bar{\phi}=180^{\circ}$, the levels on the cylinder can be up to $40 \mathrm{~dB}$ lower compared to the near side of the cylinder. In fact, on the near side of the cylinder, around $\bar{\phi}=0$, the levels on the cylinder are around $6 \mathrm{~dB}$ higher compared without the cylinder; this is due to pressure doubling.

On examining how the sound field varies along the axial extent of the cylinder, the maximum SPL is seen to be located close to the source plane, but not at $\bar{z} / a_{0}=0$. The maximum SPL would be at $\bar{z} / a_{0}=0$ if the mode angle was equal to $90^{\circ}$, but all the cut-on modes have mode angles less than $90^{\circ}$. In fact, the mode angles associated with the rotor-locked modes are, typically, in the range between $70^{\circ}$ to $80^{\circ}$. Hence the maximum SPL will be near the source plane, but further upstream the levels can fall rapidly with axial distance owing to the rapidly falling level of the rotor-locked modal component of the pressure field, as shown in Fig. 13(b) \& (c).

\section{v. CONCLUSIONS}

A new theoretical solution has been derived which predicts the acoustic pressure on a cylinder fuselage due to a disc source located adjacent to the cylinder. The distributed source is specified in terms of a spinning mode in a cylindrical duct. Hence this model can be used for single-mode calculations of the pressure on a cylindrical fuselage owing to a fan tone radiated from a turbofan intake duct. The methodology follows similar analysis which has been published previously for a point source adjacent to a cylinder. This type of solution also has been derived for source models for an open rotor. (These use rotating point or distributed source models.)

Illustrative examples show how this model can be used to predict the near-field acoustic pressure. Comparison with experimental measurements show the importance of simulating a multi-mode source in order to correctly predict the near-field pressure. Thus it is inferred that the pressure on the fuselage also should be predicted by modelling fan tones using multimode sources. This is of particular relevance for supersonic 


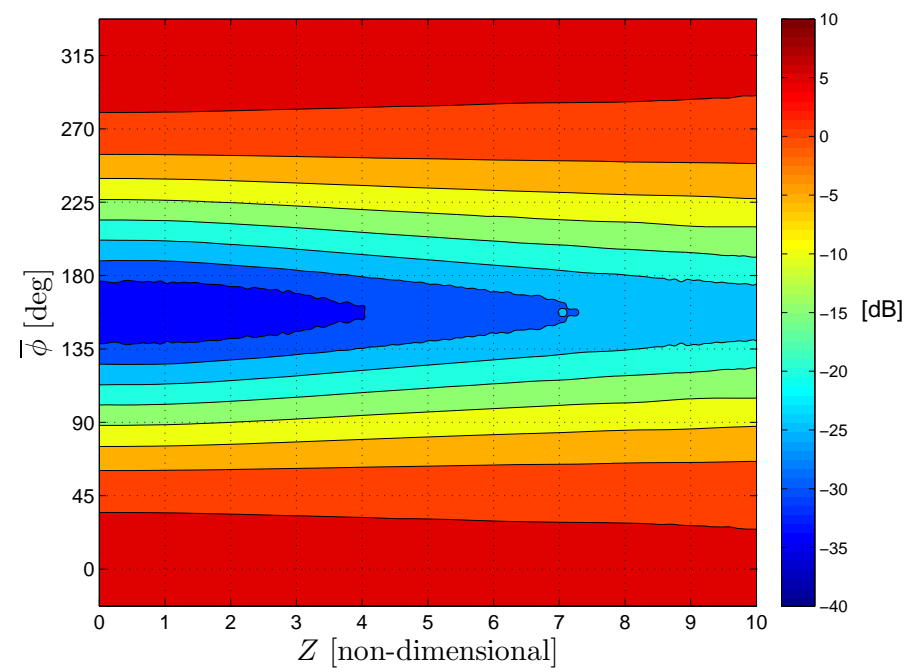

FIG. 15. Contour plot of $\Delta=\mathrm{SPL}_{\text {with cylinder }}-\mathrm{SPL}_{\text {no cylinder. }}$ Simulation at blade passing frequency, $90 \%$ fan speed. The source is taken to be equal energy per mode. (Color online)

fan tones, notwithstanding the fact that the rotor-locked component of the pressure field is likely to be dominant.

Future work will investigate the effect of the fuselage boundary layer, using theoretical methods. This problem recently also has been investigated by Dierke et al. ${ }^{11}$ using computational aeroacoustics. Additionally, future work will investigate whether the theoretical solution of the incident field due to a spinning mode radiated from an unflanged duct can be used instead of the disc source.

\section{Acknowledgments}

The authors wishes to acknowledge the continuing financial support provided by Rolls-Royce plc through the University Technology Centre in Gas Turbine Noise at the Institute of Sound and Vibration Research.

1 J. Tyler and T. Sofrin, “Axial flow compressor noise studies", SAE Trans. 70, 309-332 (1962).

2 S. Hocter, "Exact and approximate directivity patterns of the sound radiated from a cylindrical duct", J. Sound Vib. 227, 397407 (1999).

3 J. Bowman, T. Senior, and P. Uslenghi, eds., Electromagnetic and Acoustic Scattering by Simple Shapes (North-Holland Publishing Co. - Amsterdam, 1969), Chap. 2, Sec. 2.5.2, pp. 126-127.

${ }^{4}$ C. Fuller, "Free-field correction factor for spherical acoustic waves impinging on cylinders", AIAA Journal 27, 1722-1726 (1989).

5 A. McAlpine and M. Kingan, "Far-field sound radiation due to an installed open rotor", International Journal of Aeroacoustics 11, 213-245 (2012).

6 D. Hanson and B. Magliozzi, "Propagation of propeller tone noise through a fuselage boundary layer", Journal of Aircraft 22, 63-70 (1985).

7 M. Abramowitz and I. Stegun, Handbook of Mathematical Functions (Dover Publications, inc., New York, 1965), Chap. 9, page 363.
${ }^{8}$ G. Gabard and R. Astley, "Theoretical model for sound radiation from annular jet pipes: far- and near-field solutions", J. Fluid Mech. 549, 315-341 (2005).

${ }^{9}$ G. Homicz and J. Lordi, "A note on the radiative directivity patterns of duct acoustic modes", J. Sound Vib. 41, 283-290 (1975).

10 R. Sugimoto, P. Murray, A. McAlpine, and R. Astley, "Prediction of in-duct and near-field noise for a fan rig intake", AIAA 20132022 (2013), Proceedings of the 19th AIAA/CEAS Aeroacoustics Conference, Berlin, Germany, May 27-29, 11 pages.

11 J. Dierke, R. Ewert, J. Delfs, C. Stoehr, and M. Rose, "The effect of a boundary layer on engine noise propagating to the fuselage at flight conditions", AIAA 2013-2006 (2013), Proceedings of the 19th AIAA/CEAS Aeroacoustics Conference, Berlin, Germany, May 27-29, 14 pages. 